\title{
Another step towards understanding recovery?
}

INVITED COMMENTARY ON... SELF-DETERMINATION THEORY

\section{Pat Abbott}

\begin{abstract}
Self-determination theory has been offered as a potential theoretical framework for recovery. It has been argued that a concept as wide-ranging as recovery seems likely to require a number of theoretical frameworks, including self-determination theory, which appears to be particularly applicable to the clinical and social dimensions of this concept. With its emphasis on social competence and environmental support, self-determination theory may be particularly useful as a framework for considering recovery in client groups with higher levels of service need associated with disability or risk.
\end{abstract}

Mancini (2008, this issue) offers self-determination theory as a potential theoretical framework for the recovery paradigm. Self-determination theory is a theory of human motivation and needs fulfilment based on the premise that human beings have high levels of intrinsic motivation from the earliest stages of life. This may be fostered or undermined by social and contextual conditions. Mancini argues that key factors identified within self-determination theory provide potential markers for recovery and measures for evaluating therapeutic programmes in terms of recovery orientation.

\section{Does self-determination theory offer a new way to understand and measure recovery?}

Self-determination theory represents a body of empirical evidence that identifies key factors, particularly competence, autonomy and relatedness, necessary for psychological well-being (Ryan \& Deci, 2000). Mancini argues that these factors are also central elements of recovery. It could be inferred from evidence base regarding self-determination theory that interventions that enhance competence, autonomy and relatedness should improve the recovery orientation and effectiveness of therapeutic programmes. The self-determination theory framework would appear to fit best with the clinical and social dimensions of recovery identified by Roberts
\& Wolfson (2004). The personal and existential dimensions such as hope, meaning, purpose and social justice, which are prominent within the narrative recovery literature, are not emphasised within this framework. It seems increasingly likely that a paradigm as complex and wide-ranging as recovery may require a number of frameworks to underpin its application and investigation, but selfdetermination theory could be one of them.

\section{Competence and autonomy}

Mancini's article reminds us of the importance of the environment, including physical, social and emotional components, in the fostering of psychological well-being and recovery. This is a fundamental principle of psychiatric rehabilitation (Anthony et al, 2004). Competence and autonomy may be considered to be intrinsic characteristics of an individual. Yet appropriate environmental support may considerably enhance both. As individuals, we may function competently and autonomously within our chosen environments, by virtue of acquired living skills and massive social and environmental supports (including whole chains of supply meeting our basic requirements). If these environmental supports were to be withdrawn, how competent and autonomous would we be? Individuals with longerterm severe mental illness associated with high levels of disability may require substantial environmental supports to achieve their chosen lifestyles. These

Pat Abbott is a consultant rehabilitation psychiatrist with Mersey Care NHS Trust (Ashworth Hospital, Parkbourn, Maghull, Merseyside L31 1HW, UK. Email: pat.abbott@merseycare.nhs.uk). She has worked in community rehabilitation and low secure services, before working in high security. She is a member of the Executive Committee of the Royal College of Psychiatrists' Faculty of Rehabilitation and Social Psychiatry. 
supports can be viewed as enhancing rather than undermining their autonomy and competence, in the same way that this is recognised for physical disability (Steinfeld \& Scott Danford, 1999). Selfdetermination theory, and the principles it shares with rehabilitation, provides a potentially useful framework for conceptualising recovery for people with significant ongoing disability.

\section{Relatedness and social functioning}

In addition to the practical environmental supports that are essential to our day-to-day functioning, the emotional supports that enable us to live a 'real life' as opposed to an 'alive existence' are just as crucial. The notion of living a 'real life' would appear to be central to recovery (Deegan, 1988). This is where relatedness, Mancini's third factor, enters the frame. As well as an environment that fosters autonomy and competence, we need to develop relationships that are socially and emotionally sustaining. These relationships should foster hope and self-belief and build on strengths, also key elements of recovery. The recovery literature emphasises the importance of relationships with family, friends and peers rather than with professionals. Yet there is increasing evidence that relationships with professionals that are based on partnership and collaboration may be very helpful (Priebe et al, 2005). Mancini reminds us that, for many people with severe mental illness, the treatment setting and care system can be 'a source of genuine support and an important component of the recovery process'.

Davidson et al (2006) has highlighted problems in the application of the recovery paradigm not just for those with serious ongoing illness and disability, but also for those posing significant risk or requiring involuntary treatment. The self-determination theory framework, with its emphasis on social functioning, social integration and environmental support, may be useful for such individuals, with higher levels of service need.

\section{Motivation}

Central to self-determination theory is the principle that human beings are intrinsically motivated if provided with the right conditions. Yet in a significant number of individuals with longer-term severe mental illness, both biological and psychosocial factors can impair motivation. For example, cognitive deficits, which can have a profound impact on motivation, are an important cause of poor outcome in schizophrenia (Green, 2006). Individuals with motivational problems may need carefully designed supports, developed in collaboration with them, to harness their intrinsic motivation. Coercion and reward systems may not be the answer. Mancini's article provides a valuable reminder that, according to the evidence base for self-determination theory, treatment programmes reliant on contingencies or powerful elements of control or coercion may be less likely to be successful. This is relevant not just for intensive in-patient programmes for people with severe challenging behaviour, but also when considering other interventions that use rewards, for example to improve adherence to treatment within the community.

\section{The ROPI}

In relation to the measurement of recovery, it has been acknowledged that the study of this concept is in its early stages, although a number of instruments are being developed or are already in use (Ralph et al, 2000). Mancini briefly describes some existing instruments before introducing an organisational measure based on self-determination theory, the Recovery-Oriented Practices Index (ROPI). In what can hardly be described as an overcrowded field, new additions must be considered welcome. However, the usefulness of this instrument in terms of predicting improved recovery outcome within services is still being evaluated.

\section{Professional and service responses to recovery: does self-determination theory help?}

We may continue to grapple with what the recovery paradigm really means, not just for service users themselves, but in terms of the way we practise and operate our services. Mancini uses the three core principles of self-determination theory as a basis for the identification of three theoretical service styles. In the UK, many clinicians working with clients with severe mental health difficulties may feel that they operate within services in the 'traditional/ paternalistic' category (with sometimes inevitable 'controlling' tendencies). Issues of disability and risk may be serious limiting factors in terms of promoting maximum autonomy for some service users. A first step may be to consider how we can work more collaboratively to support them to develop not just increased self-efficacy and self-determination, but also the personal responsibility that goes with these. This includes, of course, responsibility for the impact of their actions on others. Whether in the community or within the range of residential and in-patient settings, we can consider how the environment, both therapeutic and non-therapeutic elements, can enhance service users' intrinsic motivation towards these aims. 
Both mental health professionals and service users must recognise the importance of intrinsic motivation. Self-determination theory evidence supports the view that human beings must experience their behaviour to be self-determined for their intrinsic motivation to come into play. Rewards contingent on task performance undermine intrinsic motivation (Deci et al, 1999). However, most behaviours, even positive ones, are not intrinsically motivated but may still be self-determined and self-regulated. This is the case for the majority of socialised behaviours that we exhibit in day-to-day life. We may do our laundry not because we are motivated to do so for the inherent satisfaction of the task but because we need clean clothes. This task is externally motivated, although we retain a sense of choice and autonomy, which would not be the case if we were being ordered to do it. In encouraging service users to enhance their independence (or to accept treatment or avoid illegal substances), we are aiming for the outcome whereby extrinsically motivated activities that will enhance their prospects of recovery are internalised and viewed by the person as a choice congruent with their autonomy. This process is relevant, not just in terms of overcoming disability, but also in terms engaging service users in a collaborative way to make positive lifestyle choices or manage their own risk.

Mancini's article highlights the fact that recovery is being promoted internationally as a paradigm for mental health services. Principles of selfdetermination theory would suggest that mental health professionals will adopt recovery-oriented practice if it is viewed as a 'top-down' initiative. As professionals, we would be more likely to be motivated to employ recovery-oriented practice if we have moved through the process of consciously valuing it and owning it. This involves a fundamental shift towards the sharing of power and responsibility with service users (Jacobson \& Curtis, 2000). It will require not just acceptance and acknowledgement of the theory, but also the development of practical methods to promote recovery within our services (Lester \& Gask, 2006).
In my view, by conceptualising recovery within an empirically based, theoretical framework, as well as by identifying specific elements that we can recognise and support in our work with individuals and our development of services, Mancini's article has contributed to enhancing our understanding of this complex concept.

\section{Declaration of interest}

None.

\section{References}

Anthony, W., Cohen, M., Farkas, M., et al (2004) Psychiatric Rehabilitation (2nd edn). Center for Cognitive Rehabilitation, Boston University.

Davidson, L., O'Connell, M., Tondora, J., et al (2006) The top ten concerns about recovery encountered in mental health system transformation. Psychiatric Services, 57, 640-645.

Deci, E. L., Koestner, R. \& Ryan, R. M. (1999) A meta-analytic review of experiments examining the effects of extrinsic rewards on intrinsic motivation. Psychological Bulletin, 125, 627-688.

Deegan, P.E. (1988) Recovery: the lived experience of rehabilitation. Psychosocial Rehabilitation Journal, 11 (4), 11-19.

Green, M. F. (2006) Cognitive impairment and functional outcome in schizophrenia and bipolar disorder. Journal of Clinical Psychiatry, 67 (suppl. 9), 3-8.

Jacobson, N. \& Curtis, L. (2000) Recovery as a policy in mental health services: strategies merging from the States. Psychiatric Rehabilitation Journal, 23, 333-341.

Lester, H. \& Gask, L. (2006) Delivering medical care for patients with serious mental illness or promoting a collaborative model of recovery? British Journal of Psychiatry, 188, 401-402.

Mancini, A. D. (2008) Self-determination theory: a framework for the recovery paradigm. Advances in Psychiatric Treatment, 14, 358-365.

Priebe, S., Watts, J., Chase, M., et al (2005) Processes of disengagement and engagement in assertive outreach patients: qualitative study. British Journal of Psychiatry, 187, 438-443.

Ralph, R. O., Kidder, K. \& Phillips, D. (2000) Can We Measure Recovery? A Compendium of Recovery-related Instruments. Evaluation Center, Human Services Research Institute \& US Department of Health \& Human Services (available from http:/ / www.tecathsri.org)

Roberts, G. \& Wolfson, P. (2004) The rediscovery of recovery: open to all. Advances in Psychiatric Treatment, 10, 37-48.

Ryan, R. M. \& Deci, E. L. (2000) Self-determination theory and the facilitation of intrinsic motivation, social development and well-being. American Psychologist, 55, 68-78.

Steinfeld, E. \& Scott Danford, G. (1999) Enabling Environments: Measuring the Impact of Environment on Disability and Rehabilitation. Kluwer Academic/Plenum Publishers. 